\title{
Bone Marrow-derived Endothelial Progenitor Cells Are Associated with Bone Mass and Strength
}

\author{
Qun Cheng, Shangjin Lin, Bo Bi, Xin Jiang, Hongli Shi, Yongqian Fan, Weilong Lin, \\ Yuefeng Zhu, and Fengjian Yang
}

ABSTRACT. Objective. Blood vessels of bone are thought to influence osteogenesis of bone. No clinical studies have determined whether angiogenesis is related to bone mass and gene expression of growth factors. We compared bone marrow endothelial progenitor cells (EPC), which control angiogenesis of bone in postmenopausal women incurring fragility fracture, with osteoporosis or traumatic fracture with normal bone mass (COM).

Methods. Bone specimens were obtained from age-matched women with osteoporosis or COM. Mononuclear cells were isolated and EPC were detected by flow cytometry. The expression levels of specific genes were measured. Bone mineral density (BMD) was determined, and serum markers of bone turnover also were measured. Differences between OP and COM were assessed with Student t test or Mann-Whitney U test, and correlations were determined using Spearman's correlation.

Results. Compared with COM, patients with OP had significantly lower levels of serum osteocalcin, procollagen type-1 N-terminal propeptide, and 25-hydroxy vitamin D, as well as decreased BMD of total hip and femoral neck and fewer bone marrow EPC. Expression levels of vascular endothelial growth factor, angiopoietin-1 (Ang-1), angiopoietin 2 (Ang-2), and the osteoblast-specific genes runt-related transcription factor $2(R U N X 2)$ and osterix in bone were significantly lower in OP than in COM. We determined that mature EPC were correlated positively with BMD of the femoral neck and total hip, gene expression of Ang-1, RUNX2, and CD31, and negatively with gene expression of receptor activator of nuclear factor- $\kappa \mathrm{B}$ ligand and Ang-2.

Conclusion. Our results demonstrate correlations of bone marrow EPC with bone mass and gene expression of growth factors, which support a hypothesis of crosstalk between angiogenesis and osteogenesis in bone health. (First Release September 1 2018; J Rheumatol 2018;45:1696-704;doi:10.3899/ jrheum.171226)

Key Indexing Terms:

ENDOTHELIAL PROGENITOR CELL FRAGILITY FRACTURE

ANGIOGENESIS

OSTEOPOROSIS OSTEOGENESIS

From the Department of Osteoporosis and Bone Disease, Huadong Hospital, affiliated to Fudan University, Research Section of Geriatric Metabolic Bone Disease, Shanghai Geriatric Institute; the Department of Orthopedics, Huadong Hospital, affiliated to Fudan University; and the Central Laboratory, Huadong Hospital, affiliated to Fudan University, Shanghai, China.

Supported by the National Natural Science Foundation of China (No. 81471089), Shanghai Municipal Commission of Health (2013ZYJB0801), Natural Science Foundation of Shanghai China (16411954600), and Shanghai Key Laboratory of Clinical Geriatric Medicine (13dz2260700). Q. Cheng, MD, PhD, Department of Osteoporosis and Bone Disease, Huadong Hospital, affiliated to Fudan University, Research Section of Geriatric Metabolic Bone Disease, Shanghai Geriatric Institute; S. Lin, MD, Department of Orthopedics, Huadong Hospital, affiliated to Fudan University; B. Bi, MD, PhD, Central Lab, Huadong Hospital, affiliated to Fudan University; X. Jiang, MD, Department of Osteoporosis and Bone Disease, Huadong Hospital affiliated to Fudan University, Research Section of Geriatric Metabolic Bone Disease, Shanghai Geriatric Institute; H. Shi, MD, Department of Osteoporosis and Bone Disease, Huadong Hospital, affiliated to Fudan University, Research Section of Geriatric Metabolic Bone Disease, Shanghai Geriatric Institute; Y. Fan, MD, PhD, Department of Orthopedics, Huadong Hospital, affiliated to Fudan University; W. Lin, MD, Department of Orthopedics, Huadong Hospital, affiliated to Fudan University; Y. Zhu, MD, Department of Orthopedics, Huadong Hospital, affiliated to Fudan University; F. Yang, MD, PhD, Department of Orthopedics, Huadong Hospital, affiliated to Fudan University. Qun Cheng and Shangjin Lin are co-first authors of this article.

\author{
Address correspondence to Dr. Q. Cheng, Department of Osteoporosis and \\ Bone Disease, Huadong Hospital, affiliated to Fudan University, Research \\ Section of Geriatric Metabolic Bone Disease, Shanghai Geriatric \\ Institute, 221 West Yan An Road, Shanghai 200040, China. \\ E-mail: quncheng_2014@163.com \\ Accepted for publication May 18, 2018.
}

Osteoporosis has become a serious challenge in China, which has the largest population in the world and an increasing proportion of elderly individuals ${ }^{1}$. The prevalence of osteoporosis in central China is about $14.2 \%$ among people more than 40 years of age and $37.5 \%$ among older women ${ }^{2}$. The rate of osteoporotic fractures has dramatically increased, with low-energy hip fractures constituting the most severe complications. These fractures could be lethal, have increased by about $10 \%$ per year, and affect $15.9 \%$ of women and $14.3 \%$ of men aged 70 years or older ${ }^{3}$.

Low-energy fractures are associated with low bone mass and poor bone architecture, which can result from pathologic conditions such as senile osteoporosis. Reduction in skeletal growth factors may yield senile osteoporosis, but the exact etiology of this disease is unclear. Studies have suggested that

Personal non-commercial use only. The Journal of Rheumatology Copyright @ 2018 . All rights reserved. 
decreased blood flow in bone may constitute a pathology of senile osteoporosis ${ }^{4}$. In our previous study, we found that a decrease in bone blood perfusion was relevant to age-related bone $\operatorname{loss}^{5}$. Kusumbe, et al identified a distinct capillary subtype (type $\mathrm{H}$ ), characterized by elevated expression of the markers CD31 and endomucin (EMCN); it couples angiogenesis and osteogenesis in young mice. Type $\mathrm{H}$ capillaries are located near the metaphysis and endosteum of the long bone and are decreased in number and size in aged animals, thereby reducing osteogenesis ${ }^{6}$. However, type $\mathrm{H}$ capillaries have not been detected with specific markers in human bone tissue. Moreover, the function of type $\mathrm{H}$ capillaries in angiogenesis and osteogenesis is still unknown.

In our current study, we measured endothelial progenitor cells (EPC) in bone marrow and determined the cytokines in the bone metaphysis of elderly women with fragility fracture or with traumatic fracture. We also detected bone mass and bone turnover markers in these patients to illustrate the relationship of bone marrow-derived EPC with bone mass, bone metabolism, and cytokines in bone tissue. Our findings support the possibility of a relevant relationship between bone strength and the number of bone marrowderived EPC. Low bone mass and fragility fractures were associated with a decreased number of EPC, especially mature ones; however, the number of EPC was positively correlated with angiogenesis and osteogenesis in the bone in elderly women.

\section{MATERIALS AND METHODS}

Ethics statement. This study was approved by the Ethical Committee of Huadong Hospital, which is affiliated with Fudan University (Project No. 2014K004). All participants gave written informed consent.

Patients and study design. A total of 118 elderly women were consecutively recruited from the department of orthopedics of Huadong Hospital from November 2014 to December 2016. Of these, 73 patients (65-92 years; 35 hypertension, 14 diabetes, 10 coronary heart disease) who had incurred a low-energy hip fracture were enrolled in the osteoporosis group (OP). Forty-five age-matched patients (68-89 years; 22 hypertension, 9 diabetes, 6 coronary heart disease) with traumatic proximal femur fractures that necessitated hip arthroplasty surgery were recruited as the comparison group $(\mathrm{COM})$. All the patients underwent arthroplasty within $48 \mathrm{~h}$ after fracture, and all the comparison patients were included in the study only if the bone mineral density (BMD) test results of both lumbar spine and hip were in the normal range $(>-1.0 \mathrm{SD}$ according to the World Health Organization criteria $\left.^{7}\right)$.

All patients were free of other systemic diseases with severe bone metabolic effects, and all subjects were taking no medication that could influence bone metabolism.

Biochemical markers of bone turnover. Biochemical markers of bone turnover were measured in all patients on the day of surgery. Blood samples were collected from $8 \mathrm{AM}$ to $10 \mathrm{AM}$ after an overnight fast. Bone formation markers included N-terminal propeptide of type-1 collagen (P1NP; Roche) and serum osteocalcin [i.e., bone Gla protein (BGP); Roche]. Bone resorption markers included collagen cross-linked C-telopeptide (CTX; Roche). The within-run and between-run intraassay coefficient of variability (CV) was $<6 \%$ for P1NP, $<6 \%$ for CTX, and $<7 \%$ for BGP. Serum PTH and 25-hydroxy vitamin $\mathrm{D}(25(\mathrm{OH}) \mathrm{D})$ were measured with enzyme immunoassays (Roche; within-run intraassay $\mathrm{CV},<3.4 \%$; and between-run intraassay $\mathrm{CV},<7 \%)$.
Measurement of BMD. BMD were determined in the contralateral total hip, femoral neck, and lumbar spine (L1-L4) by dual-energy x-ray absorptiometry (Hologic Discovery).

Collection of bone specimens. Trabecular bone cores from the intertrochanteric region of the proximal femur were obtained from patients during hip arthroplasty surgery as described previously (Supplementary Figure 1, available from the authors on request). The intertrochanteric region was chosen because it is not exactly the fracture site and is readily available during arthroplasty ${ }^{8}$

Isolation of EPC, osteoblasts, and flow cytometry. Trabecular bone cores isolated from surgery using RNAse-free instruments were crushed with a mortar and pestle in a medium of phosphate buffered saline (PBS). Supernatant from the bone wash was collected and filtered through $150 \mu \mathrm{m}$ nylon mesh, and the bone chips were further processed as described below. The supernatant that was washed from the bone marrow was processed by density gradient centrifugation to isolate the mononuclear cell layer. The supernatant was diluted with an equal volume of lymphocyte separation medium (Axis-Shield) at $37^{\circ} \mathrm{C}$ and then was subjected to density gradient centrifugation at $500 \mathrm{~g}$ for $35 \mathrm{~min}$. Mononuclear cell layers were removed and washed 3 times with PBS and then were resuspended in $100 \mathrm{ml}$ of PBS. The isolated cells were stained with the following fluorescence-conjugated antibodies: FITC (Diaclone), KDR-Alexa Flour (Biolegend), and CD133-phycoerythrin (Miltenyi Biotec). EPC were divided into 4 populations on the basis of surface antibody expression: (1) CD34+/KDR+/CD133-; (2) $\mathrm{CD} 34+/ \mathrm{CD} 133+/ \mathrm{KDR}+$; (3) $\mathrm{CD} 34+/ \mathrm{CD} 133-/ \mathrm{KDR}-$; and (4) CD34+/CD133+/KDR-. After appropriate gating, the numbers of the 4 EPC populations were quantified. The CD34+ cells were collected for further cell co-culture.

Bone chips were incubated with collagenase (Roche Applied Science) for $30 \mathrm{~min}$ at $37^{\circ} \mathrm{C}$ as the digestion. After the digestion, the solution was centrifuged and suspended cells were collected. Cells were incubated with a human alkaline phosphatase (AP) biotinylated antibody (R\&D Systems) for $30 \mathrm{~min}$ and then incubated with antibiotin microbeads (Miltenyi Biotec) for $15 \mathrm{~min}$ and positively selected by autoMACS (Miltenyi Biotec). The $\mathrm{AP}+$ cells were collected for further cell co-culture.

EPC/osteoblast noncontact co-culture conditions. The effect of EPC paracrine signaling on osteoblastic activity was investigated using a noncontact transwell co-culture model. Osteoblasts were seeded at a density of $1 \times 10^{5}$ cells per well into standard 6-well culture dishes and grown to confluency. EPC were seeded into permeable $(0.4 \mu \mathrm{m}$-pore $)$ transwell culture inserts (Merck Millipore) at a density of $1.5 \times 10^{5} \mathrm{EPC}$ per insert and grown to confluency. Transwell inserts were then positioned into osteoblast plate wells to establish co-culture conditions. A 50:50 mixture of osteoblast:EPC growth media with $2 \%$ fetal bovine serum was used throughout the co-culture treatment period. The experimental groups were described as follows: (1) OP: osteoblasts were co-cultured with EPC both from OP; (2) COM: osteoblasts were co-cultured with EPC both from COM, and each result was controlled by the result of same group without co-culture.

RNA extraction and reverse transcription-quantitative PCR. Total RNA was isolated using TRIzol reagent (Invitrogen) with chloroform for extraction and isopropanol for precipitation.

Synthesis of cDNA was achieved from $1 \mu \mathrm{g}$ of total RNA using a cDNA synthesis kit (Takara). Quantitative real-time PCR (qRT-PCR) was performed using predesigned TaqMan Gene Expression assays. All experiments were normalized to the housekeeping gene GAPDH. The $\triangle \triangle \mathrm{Ct}$ method was used to compute gene expression values, and results are presented as fold increases relative to GAPDH expression.

Statistical analysis. Data collected included patient demographics, routine blood tests, biochemical and bone metabolic markers, BMD, populations of peripheral blood and bone marrow-derived EPC, and gene expression values. Normality of distribution was ascertained using the Student $t$ test for comparisons of 2 groups. For nonparametric distributions (EPC populations and percentages), the Mann-Whitney U test was applied. Spearman's correlation analysis was used to estimate the relationship between the EPC

Personal non-commercial use only. The Journal of Rheumatology Copyright (c) 2018. All rights reserved. 
populations, gene expression values, BMD, and other variables. Results with a $\mathrm{p}<0.05$ were considered statistically significant. All data analyses were performed using SPSS software, version 19.0 for Windows (IBM).

\section{RESULTS}

Study population. The study population consisted of elderly women with age-matched low-energy femoral neck fracture (OP group) and traumatic proximal hip fracture (COM group) who underwent hip arthroplasty. Compared with the COM group, the OP group had significantly lower weight, body mass index (BMI), serum uric acid, serum levels of BGP and $\mathrm{P} 1 \mathrm{NP}$, and BMD of the total hip and femoral neck (Table 1). EPC from bone marrow or peripheral blood in the $O P$ and COM groups. EPC from bone marrow or peripheral blood were identified regarding mononuclear cells using antibodies against CD34, vascular endothelial growth factor receptor-2 (VEGFR2; KDR), and CD133 by flow cytometry (Supplementary Figure 2, available from the authors on request). In accordance with their initial discovery, EPC were defined as cells positive for bone marrow-derived hematopoietic stem cell (HSC) markers, such as CD34, as well as an endothelial marker protein, such as KDR. The more immature HSC marker CD133 also was used to identify EPC; this marker is expressed on HSC but is absent from mature endothelial cells. Thus, CD34+ cells reflect HSC, CD34+CD133-
VEGFR2+ cells represent mature EPC, and CD34+CD133+ VEGFR2+ cells represent immature EPC.

Within $48 \mathrm{~h}$ after fracture, the OP group had significantly lower cell quantities than did the COM group, as follows: the population of bone marrow HSC (OP: $0.51 \pm 0.35 \times 10^{6} / \mathrm{ml}$ vs COM: $\left.1.62 \pm 1.0 \times 10^{6} / \mathrm{ml}\right)$, the subpopulation of mature $\operatorname{EPC}\left(0.34 \pm 0.26 \times 10^{6} / \mathrm{ml}\right.$ vs $\left.1.19 \pm 0.69 \times 10^{6} / \mathrm{ml}\right)$, the subpopulation of immature EPC $\left(0.03 \pm 0.02 \times 10^{6} / \mathrm{ml}\right.$ vs $\left.0.07 \pm 0.04 \times 10^{6} / \mathrm{ml}\right)$, and the percentage of mature EPC $(66.27 \% \pm 21.2 \%$ vs $76.13 \% \pm 19.1 \%)$. However, the percentage of immature EPC $(6.88 \% \pm 3.1 \%$ vs $3.81 \% \pm$ $2.6 \%$ ) was significantly higher in the OP group, and no between-group difference was observed for other bone marrow EPC populations. Further, the amount of circulating EPC was similar for OP and COM groups (Table 2).

Gene expression of growth factors and cytokines implicated in angiogenesis, osteogenesis, and osteoclast activity. Gene expression of growth factors and cytokines was detected by qRT-PCR and was calculated from mRNA values normalized to $G A P D H$. The expression levels of 13 genes were assessed: 7 angiogenesis-related genes [hypoxia inducible factor ( $h i f)$, $E M C N, C D 31, V E G F-A$ and $-C$, angiopoietin $(A N G)-1$ and -2], 2 genes of the receptor activator of nuclear factor- $\kappa \mathrm{B}$ (RANK)/RANK ligand (RANKL)/ osteoprotegerin (OPG)

Table 1. Baseline characteristics of the 2 groups in the study. Data are mean \pm SD.

\begin{tabular}{|c|c|c|c|}
\hline Characteristics & Osteoporosis, $\mathrm{n}=73$ & Osteoarthritis, $\mathrm{n}=45$ & $\mathrm{p}$ \\
\hline Age, yrs & $70.37 \pm 6.5$ & $68.60 \pm 7.2$ & 0.11 \\
\hline Weight, kg & $59.41 \pm 8.5$ & $69.46 \pm 12.7$ & 0.02 \\
\hline Height, $\mathrm{cm}$ & $161.97 \pm 7.9$ & $162.68 \pm 8.3$ & 0.75 \\
\hline BMI, $\mathrm{kg} / \mathrm{m}^{2}$ & $22.68 \pm 3.1$ & $26.14 \pm 4.4$ & 0.01 \\
\hline Erythrocyte count, $10^{12} / 1$ & $4.27 \pm 0.5$ & $4.24 \pm 0.6$ & 0.88 \\
\hline Leukocyte count, $10^{9} / 1$ & $8.75 \pm 3.1$ & $6.49 \pm 1.8$ & 0.02 \\
\hline Neutrophile granulocyte, $10^{9} / 1$ & $6.96 \pm 3.1$ & $4.2 \pm 2.0$ & 0.001 \\
\hline Lymphocyte, $10^{9} / 1$ & $1.39 \pm 0.5$ & $1.81 \pm 0.9$ & 0.27 \\
\hline Monocyte, $10^{9} / 1$ & $0.57 \pm 0.2$ & $0.50 \pm 0.1$ & 0.68 \\
\hline Albumin, g/l & $36.34 \pm 4.3$ & $38.12 \pm 4.8$ & 0.13 \\
\hline Cholesterol, $\mathrm{mmol} / \mathrm{l}$ & $4.73 \pm 1.2$ & $4.9 \pm 1.2$ & 0.59 \\
\hline Triglyceride, $\mathrm{mmol} / \mathrm{l}$ & $1.21 \pm 1.1$ & $1.14 \pm 0.5$ & 0.43 \\
\hline Uric acid, $\mu \mathrm{mol} / 1$ & $254.42 \pm 86.3$ & $363.36 \pm 87.1$ & 0.001 \\
\hline Creatinine, $\mu \mathrm{mol} / 1$ & $74.21 \pm 27.4$ & $77.24 \pm 27.3$ & 0.67 \\
\hline ALP, $\mathrm{u} / \mathrm{l}$ & $78.18 \pm 25.5$ & $74.36 \pm 19.2$ & 0.53 \\
\hline Serum calcium, mmol/1 & $2.18 \pm 0.1$ & $2.23 \pm 0.1$ & 0.11 \\
\hline Serum phosphorus, $\mathrm{mmol} / \mathrm{l}$ & $1.07 \pm 0.2$ & $1.16 \pm 0.2$ & 0.08 \\
\hline Serum CTX, pg/ml & $571.54 \pm 195.8$ & $656.58 \pm 175.3$ & 0.37 \\
\hline Serum BGP, $\mathrm{ng} / \mathrm{ml}$ & $14.21 \pm 7.7$ & $21.01 \pm 9.4$ & 0.02 \\
\hline Serum P1NP, ng/ml & $42.67 \pm 18.8$ & $69.55 \pm 35.3$ & 0.005 \\
\hline Serum PTH, pg/ml & $39.21 \pm 15.9$ & $40.84 \pm 13.8$ & 0.73 \\
\hline Serum $25(\mathrm{OH}) \mathrm{D}, \mathrm{ng} / \mathrm{ml}$ & $13.80 \pm 7.6$ & $18.03 \pm 6.8$ & 0.08 \\
\hline BMD of $\mathrm{L} 1-4, \mathrm{~g} / \mathrm{cm}^{2}$ & $0.84 \pm 0.3$ & $0.91 \pm 0.2$ & 0.353 \\
\hline $\mathrm{BMD}$ of $\mathrm{FN}, \mathrm{g} / \mathrm{cm}^{2}$ & $0.53 \pm 0.13$ & $0.76 \pm 0.12$ & 0.004 \\
\hline BMD of total hip, $\mathrm{g} / \mathrm{cm}^{2}$ & $0.62 \pm 0.15$ & $0.76 \pm 0.13$ & 0.009 \\
\hline
\end{tabular}

Values in bold face are statistically significant. BMI: body mass index; ALP: alkaline phosphatase; CTX: collagen cross-linked C-telopeptide; BGP: bone Gla protein; P1NP: N-terminal propeptide of type-1 collagen; PTH: parathyroid hormone; 25(OH)D: 25-hydroxy vitamin D; BMD: bone mineral density; L1-4: lumbar spine 1-4; FN: femur neck. 
Table 2. Difference of EPC populations from bone marrow or peripheral blood between OP and OA groups. Data are mean $\pm \mathrm{SD}$

\begin{tabular}{lccc}
\hline & Osteoporosis, $\mathrm{n}=73$ & Osteoarthritis, $\mathrm{n}=45$ & $\mathrm{p}$ \\
\hline Bone marrow, $\times 10^{6} \mathrm{ml}$ & & & \\
C & $0.51 \pm 0.35$ & $1.62 \pm 1.0$ & $\mathbf{0 . 0 0 4}$ \\
C1 & $0.34 \pm 0.26$ & $1.19 \pm 0.69$ & $\mathbf{0 . 0 3 6}$ \\
C2 & $0.03 \pm 0.02$ & $0.07 \pm 0.04$ & $\mathbf{0 . 0 2 7}$ \\
C3 & $0.12 \pm 0.20$ & $0.34 \pm 0.31$ & 0.091 \\
C4 & $0.02 \pm 0.03$ & $0.02 \pm 0.04$ & 0.283 \\
C1\% & $66.27 \pm 21.2$ & $76.13 \pm 19.1$ & $\mathbf{0 . 0 4 1}$ \\
C2\% & $6.88 \pm 3.1$ & $3.81 \pm 2.6$ & $\mathbf{0 . 0 4 4}$ \\
C3\% & $23.52 \pm 23.7$ & $18.39 \pm 14.9$ & 0.208 \\
C4\% & $2.97 \pm 4.7$ & $1.89 \pm 2.2$ & 0.283 \\
Peripheral blood, $\times 10^{4} \mathrm{ml}$ & & \\
C & & $0.74 \pm 0.3$ & 0.93 \\
C1 & $0.53 \pm 0.2$ & $0.50 \pm 0.33$ & 0.30 \\
C2 & $0.33 \pm 0.23$ & $0.13 \pm 0.10$ & 0.52 \\
C3 & $0.09 \pm 0.08$ & $0.10 \pm 0.08$ & 0.71 \\
C4 & $0.09 \pm 0.04$ & $0.01 \pm 0.03$ & 0.15 \\
C1\% & $0.02 \pm 0.02$ & $68.0 \pm 23.7$ & 0.30 \\
C2\% & $11.94 \pm 23.7$ & $17.42 \pm 13.3$ & 0.52 \\
C3\% & $17.30 \pm 8.5$ & $13.06 \pm 9.4$ & 0.71 \\
C4\% & $3.96 \pm 3.1$ & $1.46 \pm 1.3$ & 0.15 \\
\hline
\end{tabular}

Comparisons were assessed by the Mann-Whitney $\mathrm{U}$ test. $\mathrm{P}$ values $<0.05$ were considered statistically significant and are in bold face. $\mathrm{C}=\mathrm{CD} 34+$ cells; $\mathrm{C} 1=\mathrm{CD} 34+\mathrm{CD} 133-\mathrm{KDR}+$ cells; $\mathrm{C} 2=\mathrm{CD} 34+\mathrm{CD} 133+\mathrm{KDR}+$ cells; $\mathrm{C} 3=\mathrm{CD} 34+/ \mathrm{CD} 133-/ \mathrm{KDR}-$ cells; $\mathrm{C} 4=\mathrm{CD} 34+/ \mathrm{CD} 133+/ \mathrm{KDR}-$ cells; $\mathrm{C} 1 \%=$ percentage of CD34+CD133$\mathrm{KDR}+$ cells from $\mathrm{C} ; \mathrm{C} 2$ = percentage of CD34+CD133+KDR+ cells from C; $\mathrm{C} 3=$ percentage of +/CD133-/KDRcells from $\mathrm{C}$; $\mathrm{C} 4$ = percentage of $\mathrm{CD} 34+/ \mathrm{CD} 133+/ \mathrm{KDR}$ - cells from C. EPC: endothelial progenitor cells; OP: osteoporosis; OA: osteoarthritis; KDR: vascular endothelial growth factor receptor-2.

system (TNFSF11, TNFRSF11B), 2 osteoblast-specific genes [RUNX2, osterix $(O S X)]$, and 2 osteoclast-specific genes (PDGFB, CTSK). The gene expression levels of VEGFA ( $\mathrm{p}=0.002)$, Ang-1 ( $\mathrm{p}=0.038)$, and Ang-2 ( $\mathrm{p}=0.041)$, which are related to angiogenesis, and $R U N X 2(\mathrm{p}=0.01)$ and $O S X$ $(\mathrm{p}=0.044)$, which are related to osteoblast activity, were significantly lower in the OP group than in the COM group. However, other genes, especially those related to osteoclast activity, showed no significant differences between groups (Figure 1).

Correlation of EPC populations with age, BMI, bone mass, and gene expression of growth factors. Correlations between EPC populations and age, BMI, bone mass, and gene expression levels were determined by Spearman's correlation analysis. CD34+ cells were positively correlated with most subpopulations of EPC $(\mathrm{r}=0.044-0.452, \mathrm{p}<0.05)$. CD34+CD133-KDR+ subpopulations (mature EPC) were significantly negatively associated with CD34+CD133+KDR+ cells (immature EPC) and CD34+CD133+KDR- subpopulations $(r=-0.290, p<0.05$; and $r=-0.530, p<0.05)$, but positively associated with BMD of the femoral neck and total hip $(r=0.847, p<0.05$; and $r=0.925, p<0.01)$. However, CD34+CD133+KDR+ cells (immature EPC) were negatively associated with BMD of the total hip $(\mathrm{r}=-0.817, \mathrm{p}<0.05$; Table 3).
Regarding the correlation of EPC populations with gene expression levels in the trabecular bone, we determined that total CD34+ cells were significantly positively correlated with VEGFA $(\mathrm{r}=0.532, \mathrm{p}<0.05)$. CD34+CD133-KDR+ subpopulations were significantly negatively correlated with RANKL ( $\mathrm{r}=-0.594, \mathrm{p}<0.05)$, Ang-2 $(\mathrm{r}=-0.262, \mathrm{p}<0.05)$, and positively correlated with Ang- $1(\mathrm{r}=0.550, \mathrm{p}<0.05)$, RUNX2 ( $\mathrm{r}=0.432, \mathrm{p}<0.05)$, and CD31 ( $\mathrm{r}=0.449, \mathrm{p}<0.05)$. CD34+CD133+KDR+ cells were significantly positively associated with RANKL $(\mathrm{r}=0.676, \mathrm{p}<0.01)$, and Ang-2 $(\mathrm{r}=0.484, \mathrm{p}<0.05 ;$ Table 4).

Co-culture of EPC and osteoblasts isolated from bone marrow in $O P$ and COM groups. To observe the direct coupling between angiogenesis and osteogenesis, the EPC and osteoblasts isolated from bone marrow of the OP or COM groups were co-cultured separately, and then mRNA for each group was extracted 5 days after co-culture. Gene expressions of EPC-specific markers [thrombomodulin (TM), von Willebrand factor $(v W F), K D R$, VEGFR1 $(F L T)]$ or osteoblast-specific markers (OPG, OSX, RANKL, RUNX2) were detected by qRT-PCR. Before comparing the difference of gene expression between the OP group and the COM group, each result was controlled by the result of the same group without co-culture. We found that, compared to the OP group, gene expressions of OPG, OSX, and RUNX2 or TM,

Personal non-commercial use only. The Journal of Rheumatology Copyright @ 2018 . All rights reserved. 


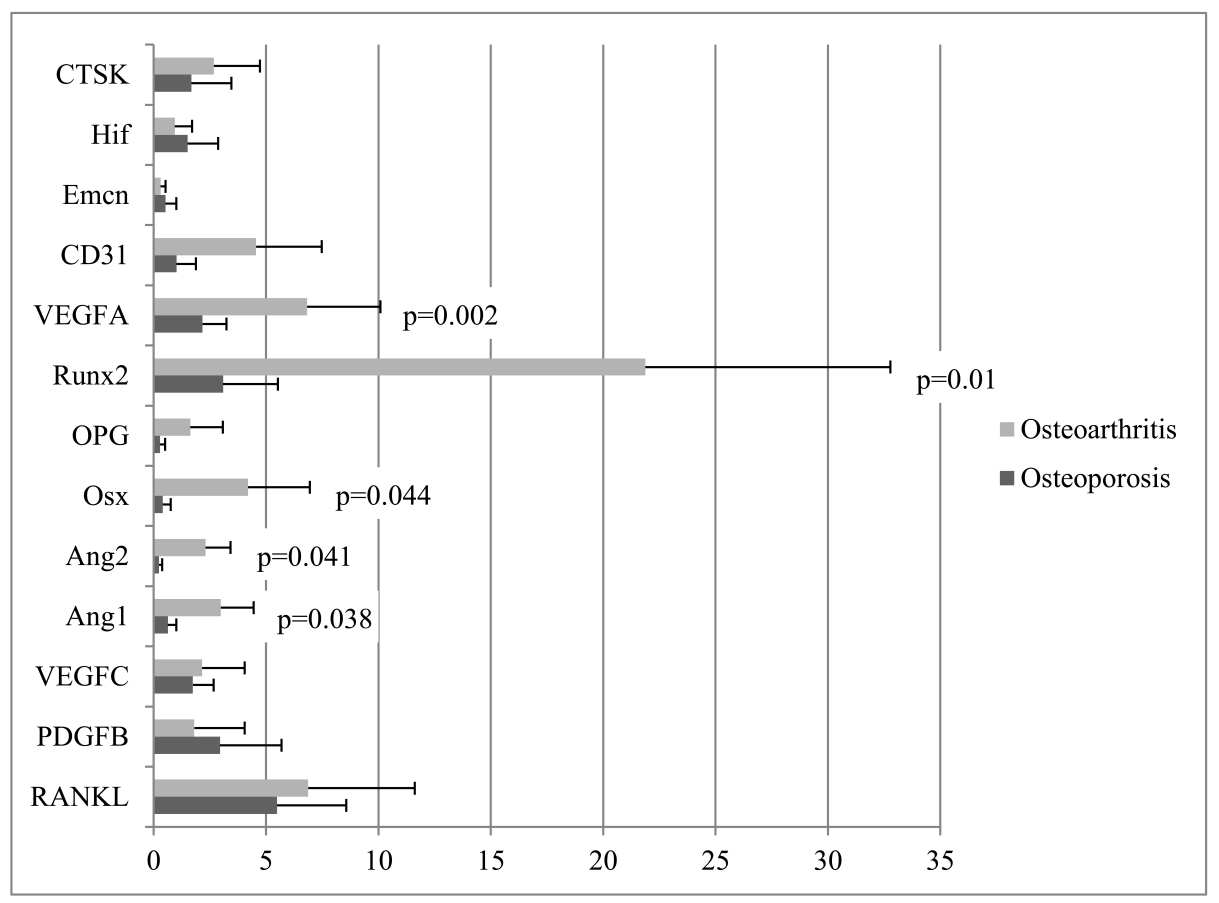

Figure 1. Comparison of gene expression of growth factors and cytokines implicated in angiogenesis and bone turnover between OP and COM. Gene expression of each growth factor or cytokine was calculated from mRNA values normalized to GAPDH mRNA value. Comparisons were assessed by the Student $t$ test. P values $<0.05$ were considered statistically significant. RANKL: receptor activator of nuclear factor- $\kappa \mathrm{B}$ ligand; PDGFB: platelet-derived growth factor B; VEGFA: vascular endothelial growth factor A; Ang-1: angiopoietin 1; Ang-2: angiopoietin 2; OSX: osterix; OPG: osteoprotegerin; RUNX2: runt-related transcription factor 2; VEGFC: VEGF C; EMCN: endomucin; HIF: hypoxia-inducible factor; CTSK: cathepsin K; OP: osteoporosis group; COM: traumatic fracture with normal bone mass.

Table 3. Correlation of EPC populations with age, BMI, and bone mass.

\begin{tabular}{|c|c|c|c|c|c|c|c|c|c|c|}
\hline & Age & BMI & $\mathrm{C}$ & $\mathrm{C} 1$ & $\mathrm{C} 2$ & $\mathrm{C} 3$ & $\mathrm{C} 4$ & L1-4 & $\mathrm{FN}$ & Total \\
\hline BMI & & 1 & -0.129 & 0.125 & 0.205 & -0.224 & -0.126 & -0.038 & 0.368 & $0.273^{*}$ \\
\hline $\mathrm{C} 1$ & & & & 1 & $-0.290 *$ & -0.528 & $-0.530^{*}$ & 0.229 & $0.847^{*}$ & $0.925^{* *}$ \\
\hline $\mathrm{C} 2$ & & & & & 1 & -0.274 & 0.209 & -0.192 & -0.710 & $-0.817 *$ \\
\hline $\mathrm{C} 3$ & & & & & & 1 & 0.282 & -0.194 & -0.684 & -0.694 \\
\hline FN & & & & & & & & & 1 & $0.931^{* *}$ \\
\hline Total & & & & & & & & & & 1 \\
\hline
\end{tabular}

Significant data are in bold face. * Correlation is significant at the 0.05 level (2-tailed). ** Correlation is significant at the 0.01 level (2-tailed). BMI: body mass index; $\mathrm{C}=\mathrm{CD} 34+$ cells; $\mathrm{C} 1=\mathrm{CD} 34+\mathrm{CD} 133-\mathrm{KDR}+$ cells; $\mathrm{C} 2=\mathrm{CD} 34+\mathrm{CD} 133+\mathrm{KDR}+$ cells; $\mathrm{C} 3=\mathrm{CD} 34+/ \mathrm{CD} 133-/ \mathrm{KDR}-$ cells; $\mathrm{C} 4=$ CD34+/CD133+/KDR- cells; EPC: endothelial progenitor cells; L1-4: BMD of lumbar spine 1-4; BMD: bone mineral density; FN: BMD of femur neck; Total: BMD of total hip.

vWF, KDR, and FLT were significantly higher in co-cultured osteoblasts or EPC in the COM group (Figure 2).

\section{DISCUSSION}

Because senile osteoporosis treatment is very challenging, there is a need for a better understanding of these cellular and molecular mechanisms underlying impaired bone meta- bolism. Therefore, we studied the populations and biological functions of bone marrow-derived EPC, and circulating EPC and the growth factors or cytokine involved in angiogenesis and bone metabolism in postmenopausal women with osteoporosis compared to those without.

We evaluated cell populations regarding the quantities of various EPC populations from bone marrow and peripheral 
Table 4. Correlation of EPC populations with growth factors and cytokines expressed in bone tissue.

\begin{tabular}{lcccccccccccccc}
\hline & RANKL & PDGFB & VEGFA & Ang-1 & Ang-2 & OSX & OPG & RUNX2 & VEGFC & CD31 & EMCN & HIF & CTSK \\
\hline C & 0.008 & -0.154 & $0.532^{*}$ & 0.287 & 0.257 & 0.239 & 0.055 & 0.194 & 0.304 & 0.218 & 0.020 & -0.054 & 0.146 \\
C1 & $-0.594^{*}$ & -0.314 & -0.353 & $0.550^{*}$ & $-0.262^{*}$ & 0.133 & 0.009 & $0.432 *$ & -0.201 & $0.449 *$ & 0.250 & -0.228 & 0.101 \\
C2 & $0.676^{* *}$ & 0.154 & 0.207 & 0.243 & $0.484^{*}$ & 0.136 & 0.161 & -0.052 & 0.134 & -0.343 & -0.150 & -0.006 & -0.168 \\
C3 & 0.005 & 0.230 & $0.549^{*}$ & -0.450 & -0.167 & -0.329 & -0.240 & -0.225 & -0.074 & -0.059 & -0.047 & -0.034 & -0.338 \\
C4 & $0.720^{* *}$ & 0.082 & -0.303 & 0.206 & 0.280 & 0.014 & 0.207 & $-0.523 *$ & 0.174 & 0.427 & -0.186 & 0.106 & -0.108 \\
\hline
\end{tabular}

* Correlation is significant at the 0.05 level (2-tailed). ** Correlation is significant at the 0.01 level (2-tailed). C = CD34+ cells; $\mathrm{C} 1=\mathrm{CD} 34+\mathrm{CD} 133-\mathrm{KDR}+$ cells; $\mathrm{C} 2=\mathrm{CD} 34+\mathrm{CD} 133+\mathrm{KDR}+$ cells; $\mathrm{C} 3=\mathrm{CD} 34+/ \mathrm{CD} 133-/ \mathrm{KDR}-$ cells; $\mathrm{C} 4=\mathrm{CD} 34+/ \mathrm{CD} 133+/ \mathrm{KDR}-$ cells; RANKL: receptor activator of nuclear factor-KB ligand; PDGFB: platelet-derived growth factor B; VEGFA: vascular endothelial growth factor A; Ang-1: angiopoietin 1; Ang-2: angiopoietin 2; OSX: osterix; OPG: osteoprotegerin; RUNX2: runt-related transcription factor 2; VEGFC: vascular endothelial growth factor C; EMCN: endomucin; HIF: hypoxia-inducible factor; CTSK: cathepsin K.

blood. EPC populations, including mature EPC, and immature EPC from bone marrow were significantly depleted in the OP group compared to the COM group. But in peripheral blood, the amounts of various EPC were similar for the 2 groups. Mature EPC were significantly positively correlated with BMD of total hip and femoral neck, whereas immature EPC were negatively correlated with BMD of cortical bone. Mature EPC from bone marrow were positively associated with cytokines of angiogenesis and osteogenesis. To our knowledge, this study is the first to demonstrate associations between various EPC in bone marrow with bone mass and cytokines in bone tissue in postmenopausal women.

Skeleton is a highly vascularized tissue ${ }^{9}$. Bone vasculature plays a vital role in bone development, modeling, and homeostasis, and bone remodeling is also tightly coupled with angiogenesis. Osteoblasts regulate bone marrow angiogenesis during bone development, and at the same time bone marrow vessels promote osteogenesis by supplementing nutrient substance and progenitor cells. Inadequate blood flow has been linked to impaired fracture healing and age-related low bone mass ${ }^{10}$. The process of vasculature development was based on studies of EPC, which can migrate from bone marrow to a developing neovasculature $^{11,12}$. EPC and osteoblast progenitor cells are spatially close to and cross-regulate each other during osteogenesis. Moreover, they are sensitive to mechanical and chemical stimulation and therefore implicated in the regulation of bone metabolism ${ }^{13}$.

EPC initially were defined as cells positive for HSC markers, such as CD34, and an endothelial marker, such as VEGFR $2^{14}$. The less mature HSC marker CD133 has also been used. The phenotype of early endothelial progenitor is CD133+/CD34+/VEGFR2+; however, loss of CD133 reflects a transformation of EPC into more mature endothelial-like cells ${ }^{13,14}$. Therefore, CD34+CD133+VEGFR2+ cells more likely represent immature progenitor cells, and CD34+CD133-VEGFR2+ cells reflect relatively mature EPC. In contrast, CD34+VEGFR2+ cells may represent cells shed from the vessel wall ${ }^{13}$.
Other investigators have reported that fractures may induce mobilization of EPC from bone marrow to the peripheral blood ${ }^{15}$. These EPC go to fracture sites and either differentiate into endothelial cells or promote fracture healing. Neovascularization begins as early as $24 \mathrm{~h}$ after traumatic fracture ${ }^{16}$, but no study reported the neovascular formation after fragility fracture, which should be retarded because anabolism is reduced with age. EPC proved to be involved in physiological and pathological neovascularization in vivo ${ }^{17}$. A study by Ma, et $a l^{18}$ indicated that the amount of immature EPC in peripheral blood increased within the first $48 \mathrm{~h}$ after fracture, peaked at 3 days, and then decreased. Mature EPC were unchanged within $48 \mathrm{~h}$ after fracture and reached their highest quantity about 5 days after fracture, then decreased to normal levels about 14 days after fracture. The numbers of various EPC types were significantly greater in younger patients than in older patients. In our study, we did not detect a significant difference in EPC subpopulations in peripheral circulation within $48 \mathrm{~h}$ after fracture for the OP versus the COM groups, whereas we found a lower level of various EPC in the bone marrow of the OP group; but the percentage of immature EPC increased in bone marrow within $48 \mathrm{~h}$ after fracture in patients with OP. The level of cell population of CD34+, which represents the level of HSC, was also lower in the OP group. However, various EPC in peripheral blood within $48 \mathrm{~h}$ post-fracture showed no significant difference. In our study, all patients were elderly, and the fractures incurred in the OP group were low-energy fractures. Moreover, the markers for EPC were not equivalent in these 2 studies. We supposed that EPC have not redistributed yet $48 \mathrm{~h}$ after fragility fracture in an elderly body because progenitor cells were exhausted, and inflammation response and stress reaction is reduced in the elderly. However, we can speculate based on the results of these studies that aging is associated with decreases in quantity and migration capacity of EPC.

Results of gene expression studies in trabecular bone indicated that the expression of some angiogenesis- and osteogenesis-related genes was significantly lower in the OP group than in the COM comparison group. Mature EPC

$$
\text { Personal non-commercial use only. The Journal of Rheumatology Copyright @ } 2018 \text {. All rights reserved. }
$$



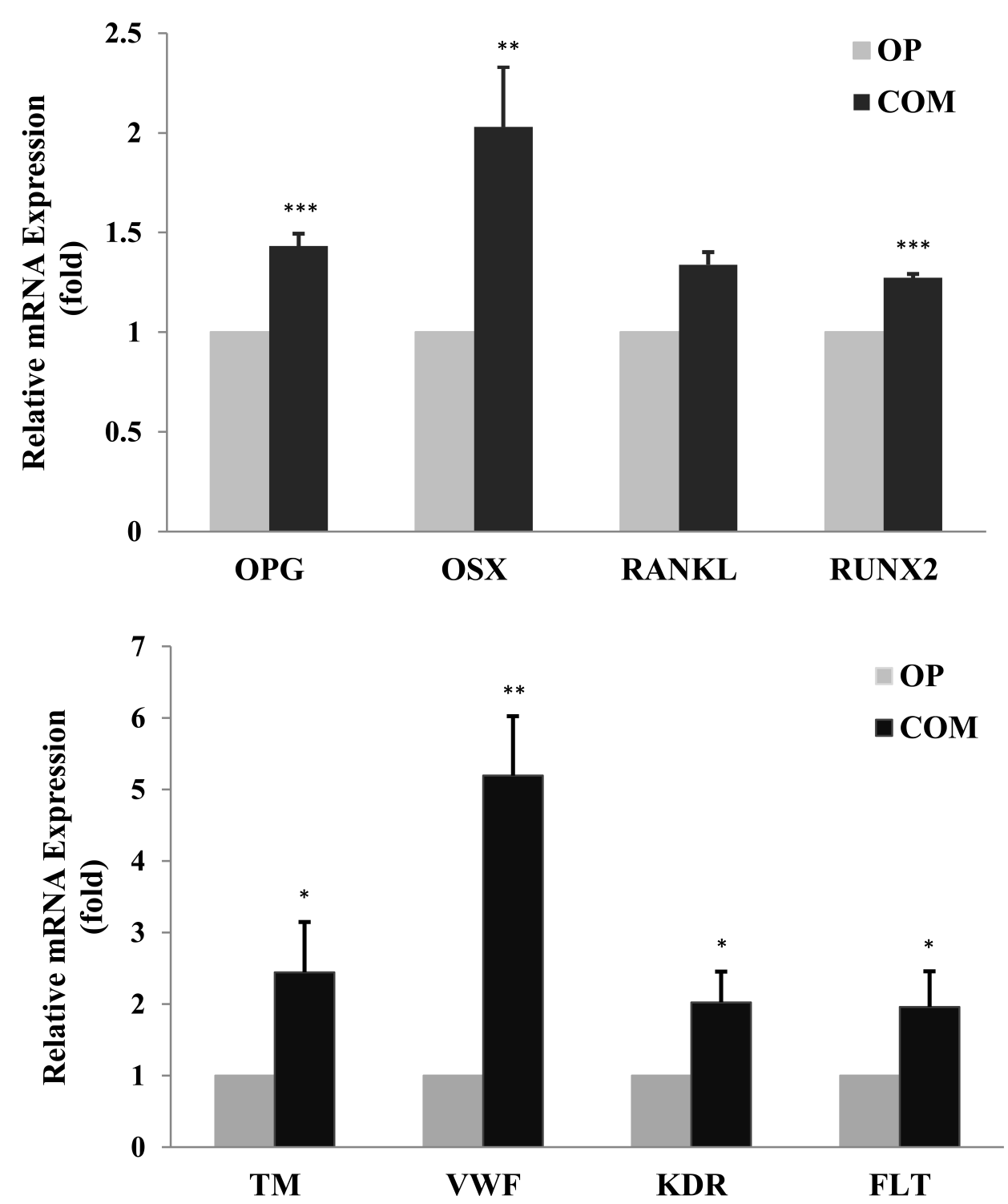

Figure 2. The expression of specific markers of osteoblast (top panel) and specific markers of EPC (lower panel) co-cultured in the OP or COM groups. Gene expression was calculated from mRNA values normalized to GAPDH mRNA value. Comparisons were assessed by the Student t test. ${ }^{*} \mathrm{p}$ values $<0.05$ were considered statistically significant. $* * \mathrm{p}<0.01$. $* *$ p $<0.001$. EPC: endothelial progenitor cells; OPG: osteoprotegerin; OSX: osterix; RANKL: receptor activator of nuclear factor- $\mathrm{\kappa B}$ ligand; RUNX2: runt-related transcription factor 2; TM: thrombomodulin; vWF: von Willebrand factor; KDR: VEGFR2 (vascular endothelial growth factor receptor 2); FLT: VEGFR1 (vascular endothelial growth factor receptor 1); OP: osteoporosis; COM: traumatic fracture with normal bone mass.

populations were significantly positively correlated with angiogenesis genes, including Ang-1, Ang-2, and CD31, and with the osteogenesis gene RUNX2. Immature EPC were significantly correlated with the inflammatory gene RANKL. We detected gene expression of CD31 and EMCN in the trabecular bone near the metaphysis, but we did not find any significant differences in the expression of mRNA between the OP and COM groups. It is possible that this crucial vessel subtype declines during aging, and the differences between our groups of elderly patients were too small to be assessed.

We further detected the gene expression of EPC and osteoblasts co-cultured in the OP group or COM group, and verified the coupling between EPC and osteoblasts in vitro. Compared to the OP group, EPC in the COM group played a stronger role in osteoblast differentiation and proliferation, which indicated the direct action of EPC on osteoblast by paracrine, and vice versa.

Accumulating molecular and clinical research evidence 
suggests that hematopoiesis and bone metabolism are interconnected ${ }^{19,20}$. In bone marrow, the production of blood cells depends upon a supportive microenvironment of hematopoietic $^{21}$ and nonhematopoietic cells ${ }^{22,23}$. Nonhematopoietic stromal cells include bone-forming osteoblasts and their precursors, which influence the differentiation of HSC into mature hematopoietic lineages, including endothelial cells ${ }^{24}$.

Our study supports the possibility of a clinically relevant relationship between bone health and functioning of EPC. Osteoporosis was associated with a lower reserve of bone marrow EPC and a maturation defect. However, after fractures in the elderly population, EPC collectively decreased and migration was hindered. This may explain why more delayed union or nonunion can be observed in the elderly. The numbers of HSC or mature EPC may be improved markers of bone mass and strength because deteriorating bone would provide a less supportive environment for angiogenesis and hematopoiesis ${ }^{25}$, resulting in a defect of EPC, which in turn lead to bone loss and delayed fracture healing. We cannot determine the initial factor based on the current cross-sectional study, but we can conclude that a close correlation exists between bone formation and vessel formation.

Our study has several strengths and limitations. The main limitation of this study is the use of patients with traumatic fracture as a comparison group rather than healthy subjects. Because we could not obtain hip samples from normal individuals, we were unable to compare our results with those in a completely normal skeleton. Instead, our comparison group was a population that had been controlled for the absence of OP. In addition, our analyses were performed only in elderly women, so the relevance of our results to other age groups or to men is unknown.

We investigated EPC populations from bone marrow and from peripheral blood and detected the gene expression of growth factors and cytokines in bone tissue involved in postmenopausal women with or without osteoporosis. We found a positive correlation between bone marrow HSC and mature EPC with bone mass and angiogenesis- or osteogenesis-related cytokines in bone tissue, which confirmed previous findings of the role of EPC in mediating crosstalk between angiogenesis and osteogenesis ${ }^{26,27}$. Our study also demonstrated that senile osteoporosis implied decreased EPC numbers and impaired maturation. Notably, the results suggested that the impairment of bone marrow EPC and bone turnover were potential mechanisms of senile osteoporosis. Therefore, osteoporotic fractures might be regarded not only as a disease caused by increased osteoclast activity, but also as an effect of reductions in osteoblast activity and blood vessel formation, especially among elderly individuals. This result might open perspectives for new osteoporosis treatments to increase or stimulate osteoblast activity and enrich bone angiogenesis instead of acting only on the inhibition of osteoclast activity.

\section{ACKNOWLEDGMENT}

We thank all participants in the study and the laboratory staff who helped to conduct the study.

\section{REFERENCES}

1. Lin X, Xiong D, Peng YQ, Sheng ZF, Wu XY, Wu XP, et al. Epidemiology and management of osteoporosis in the People's Republic of China: current perspectives. Clin Interv Aging 2015;10:1017-33.

2. Zhang Z, Shen X, Zhang H, Li S, Zhou H, Wu X, et al. The relationship between body composition and fracture risk using the FRAX model in central south Chinese postmenopausal women. Clin Endocrinol 2012;77:524-30.

3. Liu JM, Ma LY, Bi YF, Xu Y, Huang Y, Xu M, et al. A population-based study examining calcaneus quantitative ultrasound and its optimal cut-points to discriminate osteoporotic fractures among 9352 Chinese women and men. J Clin Endocrinol Metab 2012;97:800-9.

4. Ramasamy SK, Kusumbe AP, Schiller M, Zeuschner D, Bixel MG, Milia C, et al. Blood flow controls bone vascular function and osteogenesis. Nat Commun 2016;7:13601-2.

5. Naik AA, Xie C, Zuscik MJ, Kingsley P, Schwarz EM, Awad H, et al. Reduced COX-2 expression in aged mice is associated with impaired fracture healing. J Bone Miner Res 2009;24:251-64.

6. Kusumbe AP, Ramasamy SK, Adams RH. Coupling of angiogenesis and osteogenesis by a specific vessel subtype in bone. Nature 2014;507:323-8.

7. Siris ES, Adler R, Bilezikian J, Bolognese M, Dawson-Hughes B, Favus MJ, et al. The clinical diagnosis of osteoporosis: a position statement from the National Bone Health Alliance Working Group. Osteoporos Int 2014;25:1439-43.

8. Tsangari H, Findlay DM, Zannettino AC, Pan B, Kuliwaba JS, Fazzalari NL. Evidence for reduced bone formation surface relative to bone resorption surface in female femoral fragility fracture patients. Bone 2006;39:1226-35.

9. Saran U, Gemini Piperni S, Chatterjee S. Role of angiogenesis in bone repair. Arch Biochem Biophys 2014;561:109-17.

10. Laing AJ, Dillon JP, Condon ET, Street JT, Wang JH, McGuinness AJ, et al. Mobilization of endothelial precursor cells: systemic vascular response to musculoskeletal trauma. J Orthop Res 2007;25:44-50

11. Matsumoto T, Mifune Y, Kawamoto A, Kuroda R, Shoji T, Iwasaki $\mathrm{H}$, et al. Fracture induced mobilization and incorporation of bone marrow-derived endothelial progenitor cells for bone healing. J Cell Physiol 2008;215:234-42.

12. Matsumoto T, Kuroda R, Mifune Y, Kawamoto A, Shoji T, Miwa M, et al. Circulating endothelial/skeletal progenitor cells for bone regeneration and healing. Bone 2008;43:434-9.

13. Goerke SM, Obermeyer J, Plaha J, Stark GB, Finkenzeller G. Endothelial progenitor cells from peripheral blood support bone regeneration by provoking an angiogenic response. Microvasc Res 2015;98:40-7

14. Urbich C, Dimmeler S. Endothelial progenitor cells: characterization and role in vascular biology. Circ Res 2004; 95:343-53.

15. Hristov M, Erl W, Weber PC. Endothelial progenitor cells: mobilization, differentiation, and homing. Arterioscler Thromb Vasc Biol 2003;23:1185-9.

16. Stegen S, van Gastel N, Carmeliet G. Bringing new life to damaged bone: the importance of angiogenesis in bone repair and regeneration. Bone 2015;70:19-27.

17. Goligorsky MS, Salven P. Concise review: endothelial stem and progenitor cells and their habitats. Stem Cells Transl Med 2013;2:499-504.

Personal non-commercial use only. The Journal of Rheumatology Copyright @ $\odot 2018$. All rights reserved 
18. Ma XL, Sun XL, Wan CY, Ma JX, Tian P. Significance of circulating endothelial progenitor cells in patients with fracture healing process. J Orthop Res 2012;30:1860-6.

19. Valderrábano RJ, Lui LY, Lee J, Cummings SR, Orwoll ES, Hoffman AR, et al. Bone density loss is associated with blood cell counts. J Bone Miner Res 2017;32:212-20.

20. Veeriah V, Zanniti A, Paone R, Chatterjee S, Rucci N, Teti A, et al. Interleukin-1 $\beta$, lipocalin 2 and nitric oxide synthase 2 are mechano-responsive mediators of mouse and human endothelial cell-osteoblast crosstalk. Sci Rep 2016;6:29880.

21. Murayama T, Tepper OM, Silver M, Ma H, Losordo DW, Isner JM, et al. Determination of bone marrow-derived endothelial progenitor cell significance in angiogenic growth factor-induced neovascularization in vivo. Exp Hematol 2002;30:967-72.

22. Dalle Carbonare L, Valenti MT, Zanatta M, Donatelli L, Lo Cascio V. Circulating mesenchymal stem cells with abnormal osteogenic differentiation in patients with osteoporosis. Arthritis Rheum 2009;60:3356-65.
23. Zhao Q, Shen X, Zhang W, Zhu G, Qi J, Deng L. Mice with increased angiogenesis and osteogenesis due to conditional activation of HIF pathway in osteoblasts are protected from ovariectomy induced bone loss. Bone 2012;50:763-70.

24. Kuroda R, Matsumoto T, Kawakami Y, Fukui T, Mifune Y, Kurosaka M. Clinical impact of circulating CD34-positive cells on bone regeneration and healing. Tissue Eng Part B Rev 2014; 20:190-9.

25. Aqmasheh S, Shamsasanjan K, Akbarzadehlaleh P, Pashoutan Sarvar D, Timari H. Effects of mesenchymal stem cell derivatives on hematopoiesis and hematopoietic stem cells. Adv Pharm Bull 2017;7:165-77.

26. Wu L, Zhao X, He B, Jiang J, Xie XJ, Liu L. The possible roles of biological bone constructed with peripheral blood derived EPCs and BMSCs in osteogenesis and angiogenesis. Biomed Res Int 2016;2016:8168943.

27. Xie H, Cui Z, Wang L, Xia Z, Hu Y, Xian L, et al. PDGF-BB secreted by preosteoclasts induces angiogenesis during coupling with osteogenesis. Nat Med 2014;20:1270-8. 